\title{
Sphingosine 1-phosphate receptors and sphingosine kinase 1: novel biomarkers for clinical prognosis in breast, prostate, and hematological cancers
}

\author{
Susan Pyne ${ }^{1 *}$, Joanne Edwards ${ }^{2}$, Jan Ohotski ${ }^{1}$ and Nigel J. Pyne ${ }^{1}$ \\ 'Cell Biology Group, Strathclyde Institute of Pharmacy and Biomedical Sciences, University of Strathclyde, Glasgow, UK \\ ${ }^{2}$ Unit of Experimental Therapeutics, Institute of Cancer, College of Medical, Veterinary and Life Sciences, University of Glasgow, Glasgow, UK
}

\section{Edited by:}

Hans-Uwe Simon, University of Bern,

Switzerland

\section{Reviewed by:}

Deborah Stroka, University of Bern, Switzerland

Robert Friis, University of Bern, Switzerland

\section{*Correspondence:}

Susan Pyne, Cell Biology Group, Strathclyde Institute of Pharmacy and Biomedical Sciences, University of Strathclyde, 161 Cathedral Street, Glasgow G4 ORE, UK.

e-mail: susan.pyne@strath.ac.uk
There is substantial evidence for a role in cancer of the bioactive lipid sphingosine 1phosphate (S1P), the enzyme sphingosine kinase 1 (that catalyses S1P formation) and S1P-specific $G$ protein-coupled receptors. This perspective highlights recent findings demonstrating that sphingosine kinase 1 and S1P receptors are new important biomarkers for detection of early cancer and progression to aggressive cancer. The impact of the sub-cellular distribution of S1P metabolizing enzymes and S1P receptors and their spatial functional interaction with oncogenes is considered with respect to prognostic outcome. These findings suggest that S1P, in addition to being a biomarker of clinical prognosis, might also be a new therapeutic target for intervention in cancer.

Keywords: sphingosine 1-phosphate, triple negative breast cancer, estrogen receptor, disease-specific survival, recurrence

\section{INTRODUCTION}

Effective cancer therapy remains an important medical challenge. Genetic instability that leads to constitutive activating mutations of oncogenes and inactivating mutations of tumor suppressors leads to the hallmarks of cancer (Hanahan and Weinberg, 2011). It is important to identify new biomarkers that report early stage cancer or early transformation to aggressive cancer and ideally from clinical specimens. This might therefore enable early detection of cancer and inform on appropriate personalized chemotherapeutic intervention, thereby affording opportunities for improved therapies. It also follows that these biomarkers can be used to enable monitoring of effective drug intervention specific to a personalized clinical approach. Sphingosine 1-phosphate (S1P) is a bioactive lipid that has emerged as having an important role in both solid tumors and hematological cancer (Pyne and Pyne, 2010). There is now evidence that the enzymes involved in the metabolism of S1P and the cellular targets of this signaling lipid are important new therapeutically relevant biomarkers of clinical prognosis. Indeed, S1P promotes neoplastic transformation, enhances cell survival/reduces apoptosis, induces chemotherapeutic resistance, reduces senescence, promotes angiogenesis and creation of a tumor microenvironment, increases invasiveness/metastasis, and is involved in inflammation (Pyne and Pyne, 2010).

\section{SPHINGOSINE 1-PHOSPHATE METABOLISM AND ACTION IN CANCER}

Sphingosine is formed from breakdown of complex sphingolipids and is further metabolized to S1P by the enzyme, sphingosine kinase. There are two isoforms of sphingosine kinase (SK1 and SK2) that are encoded by distinct genes and differ in their biochemical properties, sub-cellular localization and functions (Pyne and Pyne, 2010). S1P can be irreversibly degraded by S1P lyase, which represents the only exit point in the sphingolipid metabolic pathway. Alternatively, S1P can be recycled to sphingosine by the action of lipid phosphate- and S1P-specific phosphatases. S1P is a bioactive lipid that binds to five different $\mathrm{G}$ protein-coupled S1P receptors (termed $\mathrm{S}_{1} \mathrm{P}_{1-5}$ ) to induce cellular responses. Intracellular S1P directly binds to TRAF2 to regulate RIP1/NFKB signaling (Alvarez et al., 2010), to prohibitin 2 to affect mitochondrial oxidative phosphorylation (Strub et al., 2011), and to $\beta$-site APP cleaving enzyme- 1 to increase amyloid- $\beta$ peptide production (Takasugi etal., 2011). So what is the relevance of S1P to cancer? A major finding that addresses this question is that SK1 mRNA transcript and/or SK1 protein expression are increased in various human tumors (Pyne and Pyne, 2010). Moreover, S1P binds to and inhibits HDAC1/2 activity to modulate histone acetylation leading to induction of immediate early genes (Hait et al., 2009). High SK1 expression in cancer cells confers positive selection to these cells, a consequence of a survival and growth advantage induced by over-expression of the enzyme. SK1 is also involved in the acquisition of replicative immortality. This is exemplified by the finding that genotoxic-induced expression of p53 in cancer cells leads to the down-regulation of SK1 expression and the induction of death by cellular senescence (Heffernan-Stroud et al., 2011). Moreover, SK1 can function as an oncogene as evidenced by the finding that over-expression of SK1 in fibroblasts induces their transformation to fibrosarcoma (Xia et al., 2000). Whereas knockdown of SK1 reduces proliferation of, for example, glioblastoma cells (Van Brocklyn et al., 2005) and androgen-independent PC-3 prostate cancer cells (Akao et al., 2006), larger, more vascularized, resistant tumors are formed when cancer cells over-expressing SK1 are injected or implanted into mice (Pyne and Pyne, 2010). In addition, SK1 
is a cellular "sensor" and confers chemotherapeutic resistance as it can promote the survival of cancer cells in the presence of anticancer agents (Loveridge et al., 2010). Therefore, targeting SK1 offers new approaches to the development of novel anti-cancer therapies.

There is also evidence for a role in cancer of S1P lyase (SPL), the enzyme that catalyses cleavage of S1P into hexadecenal and phosphoethanolamine. For example, the level of SPL mRNA expression is reduced in intestinal metastatic tumors (Oskouian et al., 2006) and the sensitivity of lung cancer cells to cisplatin and doxorubicin is increased by over-expression of SPL (Min et al., 2005). In addition, SPL is down-regulated in colon cancer, while over-expression of the enzyme induces apoptosis in HEK 293 cells (Oskouian et al., 2006). The early diagnosis of cancer is a key medical need that enhances the chances of successful treatment. In this regard, SPL is down-regulated in benign adenoma lesions of the $A p c^{M i n /+}$ mouse model, with a concomitant increase in sphingosine levels, which induces cell death (Oskouian and Saba, 2007). Cancer progression and full transformation to malignant tumors might arise from a selective pressure to increase SK1 expression to remove sphingosine and thereby promote adenoma cell survival, which might also promote neoplastic conversion. This is an example where early detection of increased expression of SK1 in the benign tumor might warrant early chemotherapeutic intervention. This seems to be borne out as knockout of the SK1 gene in a mouse model of multiple intestinal adenoma $\left(\mathrm{Apc}^{\mathrm{Min} /+}\right)$ reduces intestinal adenoma size (Kohno et al., 2006). In addition, adenomas exhibit higher levels of SK1 compared with normal mucosa and colon cancer cells that have undergone metastasis have higher expression of SK1 compared with tumors without metastasis. SK1 levels are also elevated in the azoxymethane (AOM) murine model of colon cancer. In addition, S1P levels in the blood were higher in mice with colon cancers than in those without cancers (Kawamori et al., 2009).

There is also a substantial body of evidence to demonstrate a role for S1P receptors in cancer progression. For instance, S1P stimulates migration of fibrosarcoma cells through $\mathrm{S}_{1} \mathrm{P}_{1}$ (Fisher et al., 2006) and of gastric cancer cells through $\mathrm{S}_{1} \mathrm{P}_{3}$ (Yamashita et al., 2006). Therefore, targeting SK1 and S1P receptors is an attractive approach to producing new preclinical candidates for the treatment of cancer.

\section{SPHINGOSINE 1-PHOSPHATE AS A BIOMARKER FOR CANCER DETECTION}

Recent studies have demonstrated that the circulating levels of S1P might serve as a biomarker for cancer progression. Pchejetski and colleagues have reported that circulating S1P levels are considerably lower in patients with prostate cancer compared with healthy patients and that this represents an early marker for progression to androgen-independence (Nunes et al., 2012). S1P levels also correlated with prostate-specific antigen and lymph node status. These authors also suggested that the decrease in circulating S1P during prostate cancer progression might be related to the prostate cancer-specific down-regulation of SK1 in erythrocytes and which might also account for the mechanism of cancer-induced anemia. Anemia closely follows the progression of prostate cancer. A major source for blood borne S1P is the erythrocyte and therefore circulating S1P levels are likely reduced as anemia develops and this would provide an alternative explanation of the findings.

\section{SPHINGOSINE KINASE 1 AS A BIOMARKER IN ER ${ }^{+}$BREAST CANCER}

The high tumor expression of SK1 is associated with the poor prognosis of patients with Grade 4 astrocytoma (Van Brocklyn et al., 2005). Similarly, we have reported that high tumor expression of cytoplasmic SK1 correlates with shorter disease-specific survival and recurrence time in estrogen receptor-positive $\left(\mathrm{ER}^{+}\right)$ breast cancer patients (Long et al., 2010; Watson et al., 2010). The average survival time of these patients is reduced from 18 to 7.5 years and the time to recurrence of the disease in patients receiving tamoxifen is shortened by 8 years (Long et al., 2010). However, the oncogenic background of these patients influences the clinical outcome. Indeed, when patients were stratified according to their HER1-3 status, high cytoplasmic SK1 expression in the tumors was associated with longer disease-specific survival and recurrence times (Long et al., 2010). Thus, SK1 is protective in this HER1-3 positive cancer phenotype, thereby underscoring the need to assess the effect of SK1 on clinical outcome against a variety of other disease markers as some of these can alter the signaling functionality of SK1. In this regard, we have demonstrated that SK1 is involved in promoting the survival and migration of MCF-7 ER $\mathrm{ER}^{+}$breast cancer cells (Long et al., 2010). However, stable enforced overexpression of HER2 increases SK1 mRNA and protein expression and activity in these cells and results in a decrease in the expression of HER2 in a negative feedback manner. This ablates both HER2 and S1P signaling linked with the migration of these cells (Long et al., 2010).

The sub-cellular localization of SK1 is also an important factor affecting clinical prognostic outcome. In this regard, the translocation of SK1 to the plasma membrane has been shown to be a critical determinant in neoplastic transformation (Pitson et al., 2005). However, our findings identify an additional novel role for the nuclear localization of SK1, which significantly shortens disease-specific survival and/or recurrence times in $\mathrm{ER}^{+}$breast cancer patients (Ohotski et al., 2012a). Moreover, combinations of SK1 with other signaling proteins in the same tumor have a profound effect on clinical outcome. The analysis of these associations provides evidence for potentially new S1Pdependent signaling networks in cancer cells that can be exploited therapeutically. Thus, clinical prognostic outcome is linked with the combined high expression of nuclear SK1 and cytoplasmic phosphorylated c-RAF-1 or cytoplasmic phosphorylated AKT or nuclear ERK-1/2 expression or cytoplasmic Y416 phosphorylated SFK or LYN in the same tumor (Ohotski et al., 2012a). Some of these functional associations represent well-defined interactions; for instance, SK1 has been shown to regulate AKT and this is linked with enhanced cell proliferation and the induction of chemotherapeutic resistance in various tumors (Pyne and Pyne, 2010). SK1 expression is also higher in ER negative $\left(\mathrm{ER}^{-}\right)$tumors compared with $\mathrm{ER}^{+}$breast tumors and this correlates with a poorer prognosis (Ruckhäberle et al., 2008), suggesting that expression levels of SK1 are associated with disease progression. 


\section{S1P RECEPTORS AND CLINICAL PROGNOSIS IN ER ${ }^{+}$BREAST CANCER \\ S1P 1 RECEPTOR}

The high expression of both plasma membrane $\mathrm{S}_{1} \mathrm{P}_{1}$ receptor and cytoplasmic Y216 phosphorylated c-SRC or phosphorylated c-RAF-1 in the same tumor from $\mathrm{ER}^{+}$breast cancer patients is associated with shorter recurrence time (Ohotski et al., 2012a). In addition, the high expression of both cytoplasmic $\mathrm{S}_{1} \mathrm{P}_{1}$ and ERK-1/2 or phosphorylated AKT in the same tumor is associated with shorter disease-specific survival time (Ohotski et al., 2012a). These findings suggest that the $\mathrm{S}_{1} \mathrm{P}_{1} / \mathrm{AKT}$ and $\mathrm{S}_{1} \mathrm{P}_{1} / \mathrm{ERK}-1 / 2$ modules might represent spatially restricted signaling pathways in $\mathrm{ER}^{+}$breast cancer patients that confer poor clinical prognosis by protecting cancer cells from apoptosis and/or promoting their growth/invasion.

\section{S1P $_{2}$ RECEPTOR}

In contrast with $\mathrm{S}_{1} \mathrm{P}_{1}$, high tumor nuclear expression of both c-SRC and $\mathrm{S}_{2} \mathrm{P}_{2}$ is associated with longer disease-specific survival time (Ohotski et al., 2012a). In addition, tumors with high levels of nuclear $\mathrm{S} \mathrm{P}_{2}$ receptor have significantly reduced levels of nuclear SK1, suggesting an active translocation mechanism for SK1 that is regulated by $\mathrm{S}_{\mathrm{P}} \mathrm{P}_{2}$ and accounting for its protective action in cancer patients. The $\mathrm{S}_{1} \mathrm{P}_{2}$ receptor contains a putative nuclear localization sequence that would facilitate translocation to the nucleus and where it might function to regulate nuclear signaling linked with gene expression programs. Indeed, there are a number of reports demonstrating intra-nuclear signaling by GPCR, not least the $\mathrm{LPA}_{1}$ receptor that binds LPA (Waters et al., 2006).

\section{S1P $_{\mathbf{3}}$ RECEPTOR}

High expression of both cytoplasmic LYN and cytoplasmic $\mathrm{S}_{3} \mathrm{P}_{3}$ or nuclear phosphorylated c-RAF-1 and nuclear $\mathrm{S}_{1} \mathrm{P}_{3}$ in the same tumor is associated with shorter disease-specific survival time (Ohotski etal., 2012a). In addition, high expression of both nuclear $\mathrm{S} \mathrm{P}_{3}$ and nuclear SK1 or cytoplasmic LYN and cytoplasmic $\mathrm{S}_{1} \mathrm{P}_{3}$ in the same tumor is associated with shorter recurrence time (Ohotski et al., 2012a). These represent entirely novel clinical and biochemical associations, which might constitute unique biomarker signatures to predict prognostic outcome in $\mathrm{ER}^{+}$breast cancer patients.

\section{S1P RECEPTORS AND CLINICAL PROGNOSIS}

\section{IN ER ${ }^{-}$BREAST CANCER}

We have also reported that high tumor cytoplasmic $\mathrm{S}_{\mathrm{P}} \mathrm{P}_{4}$ expression is associated with shortened disease-specific survival and recurrence times in patients with $\mathrm{ER}^{-}$tumors (Ohotski et al., 2012b). We report here for the first time the stratification of these

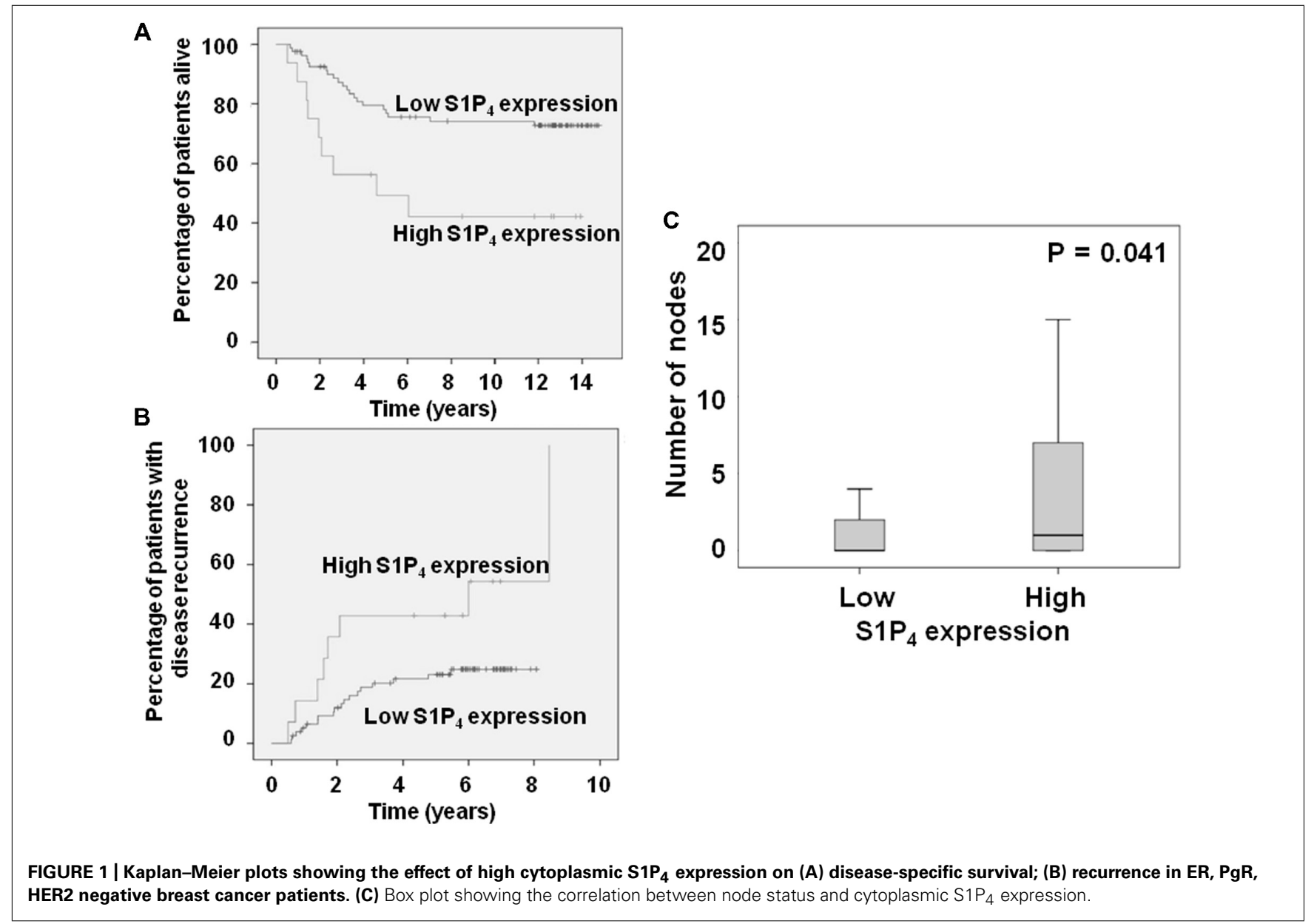


data herein to consider only patients with ER, PgR, and HER2 negative breast cancer. This analysis demonstrates that high tumor cytoplasmic $\mathrm{S}_{4} \mathrm{P}_{4}$ expression is also associated with shortened disease-specific survival and recurrence times (Figures 1A,B). High cytoplasmic $\mathrm{S}_{\mathrm{P}}$ expression is also correlated with node positive status (Figure 1C), suggesting a role for this receptor in metastasis. Mean disease-specific survival time for patients with tumors expressing high levels of $\mathrm{S} \mathrm{P}_{4}$ was 7.3 years $(n=16, \mathrm{IQR}$ 4.4-10.2) compared with 11.7 years $(n=82$, IQR $10.5-12.8)$ for the patients with tumors expressing low levels of $\mathrm{S}^{\mathrm{P}} \mathrm{P}_{4}(p=0.005)$. Mean recurrence time for patients with tumors expressing high levels of $\mathrm{S}_{4}$ was 5.1 years $(n=14, \mathrm{IQR} 3.2-7.0)$ compared with 6.6 years $(n=78$, IQR $6.0-7.2)$ for the patients with tumors expressing low levels of $\mathrm{S}_{4}(p=0.026)$. These new findings identify $\mathrm{S}_{1} \mathrm{P}_{4}$ as an important biomarker for prognostic outcome in triple negative breast cancer, and provide rationale for targeting this receptor with new chemotherapeutic anti-cancer agents.

\section{SK2 AND CANCER}

There is also new emerging evidence for an important role of SK2 in cancer. This is exemplified by the finding that siRNA knockdown of SK2 in A498, Caki-1, or MDA-MB-231 cells reduces cell proliferation and migration/invasion and this is actually more effective than knockdown of SK1 (Gao and Smith, 2011). The knockdown of SK1 or SK2 also have differential effects on p53, p21, ERK1, ERK2, FAK, and VCAM1 indicating that SK1 and SK2 have non-overlapping functions. However, to date, there have been no studies assessing the association of SK2 expression with clinical prognosis of cancer patients.

\section{MONITORING BIOMARKERS AS EVIDENCE-BASED THERAPEUTICS}

The major therapeutic intervention of S1P signaling in cancer focuses on: (i) inhibition of SK1 activity; (ii) antagonism of $\mathrm{S}_{1} \mathrm{P}_{1 / 3}$ receptors; and (iii) reduction in S1P bioavailability. Clearly, it will be important to use reliable biomarkers that provide information regarding the effectiveness of these interventions. Toward this end, we have reported that SK1 inhibitors [e.g., 2-( $p$-hydroxyanilino)4-( $p$-chlorophenyl)thiazole, $N, N$-dimethylsphingosine, and FTY720] uniquely activate the ubiquitin-proteasomal degradation pathway to remove SK1 from breast and prostate cancer cells (Loveridge et al., 2010; Tonelli et al., 2010; Ohotski et al., 2012b) This remarkable property of SK1 inhibitors, which requires an initial inhibition of SK1 activity to activate the proteasome, indicates that it is possible to create cancer cells that are SK1 null, thereby eliminating its "oncogenic" effect. The "chemical knockdown" of SK1 reduces intracellular S1P and elevates

\section{REFERENCES}

Akao, Y., Banno, Y., Nakagawa, Y., Hasegawa, N., Kim, T. J., Murate, T., et al. (2006). High expression of sphingosine kinase 1 and $\mathrm{S} 1 \mathrm{P}$ receptors in chemotherapyresistant prostate cancer PC-3 cells and their camptothecin-induced up-regulation. Biochem. Biophys. Res. Commun. 342, 12841290.
Alvarez, S. E., Harikumar, K. B., Hait, N. C., Allegood, J., Strub, G. M., Kim, E. Y., et al. (2010). Sphingosine1-phosphate is a missing cofactor for the E3 ubiquitin ligase TRAF2. Nature 465, 1084-1088.

Baran, Y., Salas, A., Senkal, C. E., Gunduz, U., Bielawski, J., Obeid, L. M., et al. (2009). Alterations of ceramide/sphingosine 1phosphate rheostat involved in the

ceramide levels, which induces apoptosis (Loveridge et al., 2010). Therefore, the "chemical knockdown" of SK1 is linked specifically with apoptosis, and may represent an important reporter for biochemical effectiveness of SK1 inhibitors in patients. The knockdown of SK1 in the tumor can be measured in biopsy samples, but monitoring in erythrocytes would be a significant advantage, although these measurements have not currently been performed.

In addition to the above, the $\mathrm{S} 1 \mathrm{P} /$ ceramide ratio in tumors is also a biomarker for effective chemotherapeutic intervention. For instance, siRNA knockdown of SK1 expression increases the sensitivity of resistant leukemic cells to imatinib (Marfe et al., 2011) and enforced expression of SK1 increases the S1P/ceramide ratio and prevents apoptosis to imatinib (Baran et al., 2009). In addition, the $\mathrm{S} 1 \mathrm{P} /$ ceramide ratio is reduced in response to imatinib in imatinib-sensitive LAMA84 cells, while the ratio is unaltered in imatinib-resistant cells. Finally, daunorubicin-sensitive but not insensitive leukemia cells (CML, AML, and ALL) exhibit a reduced $\mathrm{S} 1 \mathrm{P} /$ ceramide ratio when treated with daunorubicin and sensitivity to daunorubicin in the latter is restored by inhibiting SK1 activity (Sobue et al., 2008).

Our ability to measure the clinical effectiveness of S1P receptor modifying compounds in patients by monitoring effects directly on the S1P receptor would be a major advantage. In this regard, the cytoplasmic S1P receptor might represent a surrogate marker for receptor activation. This is based on the knowledge that S1P receptor internalization is recognized as part of the process required for signal transmission regulating gene re-programing of cancer cells. Therefore, the effectiveness of S1P receptor antagonists might be linked with reduced cytoplasmic S1P receptor levels in the tumors of patients treated with these compounds.

\section{CONCLUSION}

Clearly, the objective of S1P therapeutics is to eliminate the negative prognostic effect of S1P receptors and SK1 on disease-specific survival and recurrence in cancer patients. In addition to the expression level of these biomarkers, their activity status is also an important consideration. For instance, SK1 is activated by an ERK-1/2-specific phosphorylation of S225 in SK1 (Pitson et al., 2005). Future studies can utilize specific anti-phospho S225 SK1 antibody to determine the impact of phosphorylated SK1 on clinical prognostic significance. Moreover, specific assays that detect direct functional interaction between for instance, SK1 and an adaptor/regulatory protein using BRET and FRET technologies would enable measurement of the activation status of specific S1Pdependent signaling networks and these could then be correlated with clinical prognosis.

regulation of resistance to imatinib induced apoptosis in K562 human chronic myeloid leukaemia cells. J. Biol. Chem. 282, 1092210934.

Fisher, K. E., Pop, A., Koh, W., Anthis, N. J., Saunders, W. B., and Davis, G. E. (2006). Tumour cell invasion of collagen matrices requires coordinate lipid agonist-induced G protein and membrane-type matrix metalloproteinase-1-dependent signalling. Mol. Cancer 5, 69.

Gao, P., and Smith, C. D. (2011). Ablation of sphingosine kinase-2 inhibits tumour cell proliferation and migration. Mol. Cancer Res. 9, 15091519.

Hait, N. C., Allegood, J., Maceyka, M., Strub, G. M., Harikumar, K. B., Singh, S. K., et al. (2009). Regulation of histone acetylation in the nucleus 
by sphingosine-1-phosphate. Science 325, 1254-1257.

Hanahan, D., and Weinberg, R. A. (2011). Hallmarks of cancer: the next generation. Cell 144, 646-674.

Heffernan-Stroud, L. A., Helke, K. L., Jenkins, R. W., De Costa, A. M., Hannun, Y. A., and Obeid, L. M. (2011). Defining a role for sphingosine kinase 1 in p53-dependent tumors. Oncogene 31, 11661175.

Kawamori, T., Kaneshiro, T., Okumura, M., Maalouf, S., Uflacker A., Bielawski, J., et al. (2009). Role for sphingosine kinase 1 in colon carcinogenesis. FASEB J. 23, 405-414.

Kohno, M., Momoi, M., Oo, M. L., Paik, J. H., Lee, Y. M., Venkataraman, K., et al. (2006). Intracellular role for sphingosine kinase 1 in intestinal adenoma cell proliferation. Mol. Cell. Biol. 26, 7211-7223.

Long, J. S., Edwards, J., Watson, C., Tovey, S., Mair, K. M., Schiff, R., et al. (2010). Sphingosine kinase 1 induces tolerance to human epidermal growth factor receptor 2 and prevents formation of a migratory phenotype in response to sphingosine 1-phosphate in estrogen receptor positive breast cancer cells. Mol. Cell. Biol. 30, 3827-3841.

Loveridge, C., Tonelli, F., Leclercq, T., Lim, K. G., Long, J. S., Berdyshev, E., et al. (2010). The sphingosine kinase 1 inhibitor 2-( $p$-hydroxyanilino)4 -( $p$-chlorophenyl)thiazole induces proteasomal degradation of sphingosine kinase 1 in mammalian cells. J. Biol. Chem. 285, 3884138852 .

Marfe, G., Di Stefano, C., Gambacurta, A., Ottone, T., Martini, V., Abruzzese, E., et al. (2011). Sphingosine kinase 1 overexpression is regulated by signalling through PI3K, AKT2, and mTOR in imatinib-resistant chronic myeloid leukemia cells. Exp. Hematol. 39, 653-665.e6

Min, J., Van Veldhoven, P. P., Zhang, L., Hanigan, M. H., Alexander, H., and Alexander, S. (2005). Sphingosine-1-phosphate lyase regulates sensitivity of human cells to select chemotherapy drugs in a p38dependent manner. Mol. Cancer Res. 3, 287-296.

Nunes, J., Naymark, M., Sauer, L., Muhammad, A., Keun, H., Sturge, J., et al. (2012). Circulating sphingosine-1-phosphate and erythrocyte sphingosine kinase-1 activity as novel biomarkers for early prostate cancer detection. Br. J. Cancer 106, 909-915.

Ohotski, J., Edwards, J., Elsberger, B. Watson, C., Orange, C., Mallon, E., et al. (2012a). Identification of novel functional and spatial associations between sphingosine kinase 1, sphingosine 1-phosphate receptors and other signaling proteins that affect prognostic outcome in estrogen receptor-positive breast cancer. Int. J. Cancer. doi: 10.1002/ijc.27692 [Epub ahead of print].

Ohotski, J., Long, J. S., Orange, C. Elsberger, B., Mallon, E., Doughty, J., et al. (2012b). High expression of the sphingosine 1-phosphate receptor 4 and sphingosine kinase 1 is associated with outcome in estrogen negative breast cancer. Br. J. Cancer 106, 1453-1459.

Oskouian, B., and Saba, J. (2007). Sphingosine-1-phosphate metabolism and intestinal tumorigenesis: lipid signaling strikes again. Cell Cycle 6, 522-527.

Oskouian, B., Sooriyakumaran, P. Borowsky, A. D., Crans, A., DillardTelm, L., Tam, Y. Y., et al. (2006). Sphingosine-1-phosphate lyase potentiates apoptosis via p53- and p38-dependent pathways and is down-regulated in colon cancer. Proc. Natl. Acad. Sci. U.S.A. 103, 17384-17389.

Pitson, S. M., Xia, P., Leclercq, T. M. Moretti, P. A., Zebol, J. R., Lynn, H. E., et al. (2005). Phosphorylationdependent translocation of sphingosine kinase to the plasma membrane drives its oncogenic signalling. J. Exp. Med. 201, 49-54.

Pyne, N. J., and Pyne, S. (2010). Sphingosine 1 phosphate and cancer. Nat. Rev. Cancer 10, 489-503.

Ruckhäberle, E., Rody, A., Engels, K., Gaetje, R., von Minckwitz,
G., Schiffmann, S., et al. (2008) Microarray analysis of altered sphingolipid metabolism reveals prognostic significance of sphingosine kinase 1 in breast cancer. Breast Cancer Res. Treat. 112, 41-52.

Sobue, S., Nemoto, S., Murakami, M. Ito, H., Kimura, A., Gao, S., et al. (2008). Implications of sphingosine kinase 1 expression level for the cellular sphingolipid rheostat: relevance as a marker for daunorubicin sensitivity of leukaemia cells. Int. J. Hematol. 87, 266-275.

Strub, G. M., Paillard, M., Liang, J., Gomez, L., Allegood, J. C., Hait, N. C., etal. (2011). Sphingosine-1 phosphate produced by sphingosine kinase 2 in mitochondria interacts with prohibitin 2 to regulate complex IV assembly and respiration. FASEB J. 25, 600-612.

Takasugi, N., Sasaki, T., Suzuki, K., Osawa, S., Isshiki, H., Hori, Y., et al. (2011). BACE1 activity is modulated by cell-associated sphingosine1-phosphate. J. Neurosci. 31, 68506857.

Tonelli, F., Lim, K. G., Loveridge, C. Long, J., Pitson, S. M., Tigyi, G., et al (2010). FTY720 and (S)-FTY720 vinylphosphonate inhibit sphingosine kinase 1 and promotes its proteasomal degradation in human pulmonary artery smooth muscle, breast cancer and androgen-independent prostate cancer cells. Cell. Signal. 22, 1536-1542.

Van Brocklyn, J. R., Jackson, C. A., Pearl, D. K., Kotur, M. S., Snyder, P. J., and Prior, T. W. (2005) Sphingosine kinase-1 expression correlates with poor survival of patients with glioblastoma multiforme: role of sphingosine kinase isoforms in growth of glioblastoma cell lines. J. Neuropathol. Exp. Neurol. 64, 695-705.

Waters, C. M., Saatian, B., Moughal, N. A., Zhao, Y., Tigyi, G., Natarajan, V., etal. (2006). Integrin signalling regulates the nuclear localization and function of the lysophosphatidic acid receptor-1 (LPA1) in mammalian cells. Biochem. J. 398, $55-62$.
Watson, C., Long, J. S., Orange, C., Tannahill, C. L., Mallon, E., McGlynn, L. M., et al. (2010). High expression of sphingosine 1-phosphate receptors, $\mathrm{S}_{1} \mathrm{P}_{1}$ and $\mathrm{S}_{1} \mathrm{P}_{3}$, sphingosine kinase 1 , and extracellular signal-regulated kinase-1/2 is associated with development of tamoxifen resistance in estrogen receptor-positive breast cancer patients. Am. J. Pathol. 177, 2205-2215.

Xia, P., Gamble, J. R., Wang, L., Pitson, S. M., Moretti, P. A., Wattenberg, B. W., et al. (2000). An oncogenic role of sphingosine kinase. Curr. Biol. 10, 1527-1530.

Yamashita, H., Kitayama, J., Shida, D., Yamaguchi, H., Mori, K., Osada, M., et al. (2006). Sphingosine 1phosphate receptor expression profile in human gastric cancer cells: differential regulation on the migration and proliferation. J. Surg. Res. 130, 80-87.

Conflict of Interest Statement: The authors declare that the research was conducted in the absence of any commercial or financial relationships that could be construed as a potential conflict of interest.

Received: 29 September 2012; accepted: 28 October 2012; published online: 03 December 2012.

Citation: Pyne S, Edwards J, Ohotski J and Pyne NJ (2012) Sphingosine 1-phosphate receptors and sphingosine kinase 1: novel biomarkers for clinical prognosis in breast, prostate, and hematological cancers. Front. Oncol. 2:168. doi: 10.3389/fonc. 2012.00168

This article was submitted to Frontiers in Molecular and Cellular Oncology, a specialty of Frontiers in Oncology. Copyright (c) 2012 Pyne, Edwards, Ohotski and Pyne. This is an open-access article distributed under the terms of the Creative Commons Attribution License, which permits use, distribution and reproduction in other forums, provided the original authors and source are credited and subject to any copyright notices concerning any third-party graphics etc. 